Journal of Mathematics and Statistics 1 (3): 234-238, 2005

ISSN 1549-3644

(C) 2005 Science Publications

\title{
On the Prime Radical of a Hypergroupoid
}

\author{
Gürsel Yeşilot \\ Istanbul Technical University Fen-Edebiyat fak. Matematik Böl. 80626 Maslak, Istanbul,Turkey
}

\begin{abstract}
In this study, we give definitions of a prime ideal, a s-semiprime ideal and a w-semiprime ideal for a hypergroupoid K. For an ideal $A$ of $K$ we show that radical of $A(R(A))$ can be represented as the intersection of all prime ideals of $\mathrm{K}$ containing $\mathrm{A}$ and we define a strongly A-nilpotent element. For any ideal $A$ of $K$, we prove that $R(A)=\cap$ (s-semiprime ideals of $K$ containing $A)=\cap($ w-semiprime ideals of $K$ containing $A)=\{$ strongly $A$ nilpotent elements $\}$. For an ideal $B$ of $K$ put $B^{(0)}=B$ and $B^{(n+1)}=\left(B^{(n)}\right)^{2}$. If a hypergroupoid $K$ satisfies the ascending chain condition for ideals then $(R(A))^{(n)} \subseteq A$ for some $n$. For an ideal $A$ of $K$ we give a definition of right radical of $A\left(R_{+}(A)\right)$. If $K$ is associative then $R(A)=R_{+}(A)=R \_(A)$.
\end{abstract}

Key words: Hypergroupoids, s-semiprime ideal, w-semiprime ideal, ascending chain

\section{Hypergroupoids and Complete $\ell$-Groupoids}

Definition 1.1: A groupoid $K$ is a system $(K, \cdot)$, where $\mathrm{K}$ is a set and $\cdot$ is a binary operation on $\mathrm{K}$.

Definition $1.2^{[1]}$ : A complete $\ell$-groupoid is a system $(\mathrm{K}, \cdot)$, where $\mathrm{K}$ is a complete lattice and $\cdot$ is a binary operation on $\mathrm{K}$ which satisfies the following conditions: $\mathrm{a} \cdot \underset{\mathrm{t} \in \mathrm{T}}{\left(\mathrm{Vb}_{\mathrm{t}}\right)}=\underset{\mathrm{t} \in \mathrm{T}}{\mathrm{V}}\left(\mathrm{a} \cdot \mathrm{b}_{\mathrm{t}}\right), \underset{\mathrm{t} \in \mathrm{T}}{\left(\mathrm{Vb}_{\mathrm{t}}\right) \cdot \mathrm{a}}=\underset{\mathrm{t} \in \mathrm{T}}{\mathrm{V}}\left(\mathrm{b}_{\mathrm{t}} \cdot \mathrm{a}\right)$ for all $\mathrm{a}, \mathrm{b}_{\mathrm{t}} \in \mathrm{K}$

Let $\mathrm{K}$ be a set and denote by $2^{\mathrm{K}}$ the set of all its subsets.

Definition 1.3 ${ }^{[2]}$ : A multivariable binary operation on $\mathrm{K}$ is a map $\vartheta: K x K \rightarrow 2^{\mathrm{K}}$. A hypergroupoid is a system $(\mathrm{K}$, $\vartheta$ ), where $\mathrm{K}$ is a set and $\vartheta$ is a multivariable operation on $\mathrm{K}$.

From now on, we write $a \cdot b$ instead of $\vartheta(a, b)$

Let $(K, \cdot)$ be a hypergroupoid. For $A, B \in 2^{K} . A \neq \varnothing$, $\mathrm{B} \neq \varnothing$, put $\mathrm{A} \cdot \mathrm{B}=\cup(\mathrm{a} \cdot \mathrm{b})$ and $\varnothing \cdot \mathrm{A}=\mathrm{A} \cdot \varnothing=\varnothing$ for all $\mathrm{a} \in \mathrm{A}$ $\mathrm{b} \in \mathrm{B}$

$\mathrm{A} \in 2^{\mathrm{K}}$. Then $\left(2^{\mathrm{K}}, \cdot\right)$ is a complete $\ell$-groupoid.

Conversely, If $\left(2^{\mathrm{K}}, \cdot\right)$ is a complete $\ell$-groupoid then a restriction of the binary operation of $2^{\mathrm{K}}$ to $\mathrm{K}$ is a multivariable operation on $\mathrm{K}$ and $\mathrm{K}$ is a hypergroupoid, with respect to this operation.

Let $w$ be a ternary relation on $\mathrm{K}$.

For $(a, b) \in K x K$, put $a \cdot b=\{x \in K \mid(a, b, x) \in w\}$, then $(\mathrm{K}, \cdot)$ is a hypergrupoid.

Conversely, let $(\mathrm{K}, \cdot)$ be a hypergroupoid. Denote by $w$ the set $(a, b, c) \in K \times K \times K$ such that $a \cdot b \neq \varnothing$ and $c \in a \cdot b$. Then $w$ is a ternary relation on $K$.

Hypergroupoids contain the following two classes of algebraic systems.
1. A partial binary operation $\vartheta$ on $K$ is a map $\vartheta: A \rightarrow K$, where $A$ is a subset of $K x K$. A partial groupoid is a system $(K, \cdot)$, where $\cdot$ is a partial binary operation on $\mathrm{K}$.

Let $(K, \cdot)$ be a partial groupoid and $A$ is the definition domain of $\cdot$ For $(a, b) \notin A$ put $a \cdot b=\varnothing$. Then $\cdot$ is defined for all $(\mathrm{a}, \mathrm{b}) \in \mathrm{KxK}$ and $(\mathrm{K}, \cdot)$ is a hypergroupoid.

2. Let $\left\{k, \vartheta_{v}, v \in S\right\}$ be a universal algebra such that every $\vartheta_{\mathrm{V}}$ is a binary operation on $\mathrm{K}$. For $(a, b) \in \operatorname{KxK}$ put $a \cdot b=\left\{\vartheta_{v}(a, b), v \in S\right\}$ then $(K, \cdot)$ is a hypergroupoid.

2. Prime and Semiprime Elements of an Ordered Gruopoid: Let $(\mathrm{G}, \cdot)$ be an ordered groupoid ${ }^{[1]}$, ch XIV). An ordered groupoid $\mathrm{G}$ is called $\ell_{0}$-groupoid if $\mathrm{G}$ is a complete lattice. Denote by $1_{\mathrm{G}}$ the greatest element of $\mathrm{G}$.

Definition 2.1 ${ }^{[1]}$ : Let $(G, \cdot)$ be an $\ell_{0}$-groupoid. An element $\mathrm{p} \in \mathrm{G}$ is prime if $\mathrm{p} \neq 1_{\mathrm{G}}$ and $\mathrm{a} \cdot \mathrm{b} \leq \mathrm{p}$, for $\mathrm{a}, \mathrm{b} \in \mathrm{G}$, then $\mathrm{a} \leq \mathrm{p}$ or $\mathrm{b} \leq \mathrm{p}$.

For $a \in G, a \neq 1_{G}$, denote by $R_{G}(a)$ the intersection of all prime elements of $G$ containing a. Put $R_{G}(a)=1_{G}$ if there are not any element with this property.

Definition 2.2: An element $h \in G$ is s-semiprime if $h \neq 1_{G}$ and $\mathrm{a}^{2} \leq \mathrm{h}$, for $\mathrm{a} \in \mathrm{G}$, implies that $\mathrm{a} \leq \mathrm{h}$.

For $\mathrm{a} \in \mathrm{G}, \mathrm{a} \neq 1_{\mathrm{G}}$, denote by $\mathrm{r}_{\mathrm{G}}^{\mathrm{s}}$ (a) the intersection of all s-semiprime elements of $G$ containing a. Put $\mathrm{r}_{\mathrm{G}}^{\mathrm{s}}(\mathrm{a})=1_{\mathrm{G}}$ if there are not any element with this property. For $\mathrm{a} \in \mathrm{G}$ denote by $<\mathrm{a}>$ the groupoid generated by a. An element of the groupoid $<a>$ will be denoted by $\mathrm{f}(\mathrm{a})$.

Corresponding Author: Gürsel Yeşilot, Istanbul Technical University Fen-Edebiyat fak. Matematik Böl. 80626 Maslakl IstanbullTurkey 
Definition 2.3: An element $h \in G$ is $w$-semiprime if $\mathrm{h} \neq 1_{\mathrm{G}}$ and $\mathrm{f}(\mathrm{a}) \leq \mathrm{h}, \mathrm{a} \in \mathrm{G}, \mathrm{f}(\mathrm{a}) \in<\mathrm{a}>$ implies that $\mathrm{a} \leq \mathrm{h}$.

Therefore every w-semiprime element is ssemiprime. For $a \in G, a \neq 1_{G}$, denote by $r_{G}^{\mathrm{W}}$ (a) the intersection of all w-semiprime elements of $G$ containing a. Put $\mathrm{r}_{\mathrm{G}}^{\mathrm{W}}(\mathrm{a})=1_{\mathrm{G}}$ if there are not any element with this property. It is clear that $r_{G}^{s}(a) \leq r={ }_{G}^{W}(a) \leq R_{G}(a)$ for all $\mathrm{a} \in \mathrm{G}$.

\section{The Prime Radical of an Ideal}

Definition3.1: Let K be a hypergroupoid. A right (left) ideal of $\mathrm{K}$ is a subset $\mathrm{H}$ such that haᄃH (respectively $a \cdot h \subseteq H$ ) for all $a \in K, h \in H$. An (two-side) ideal of $K$ is a

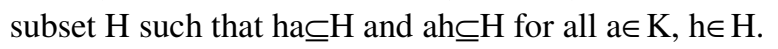
Denote by $\operatorname{Id}(\mathrm{K})\left(\operatorname{Id}_{+}(\mathrm{K})\right.$, Id_(K)) the set of all ideals (respectively, right ideals, left ideals) of K. Put $\varnothing \in \operatorname{Id}(K), \varnothing \in \operatorname{Id}_{+}(K), \varnothing \in \operatorname{Id}_{-}(\mathrm{K})$. Then $\operatorname{Id}(K), \operatorname{Id}_{+}(\mathrm{K})$, Id_(K) are complete lattices with respect to the inclusion relation.

Proposition 3.2: Let $K$ be an hypergroupoid. Then:

1. $\cap A_{t} \in \operatorname{Id}(K)$ and $\cup A_{t} \in \operatorname{Id}(K)$ for any $A_{t} \in \operatorname{Id}(K)$; $t \in T \quad A_{t \in T}$

2. $\cap B_{t} \in \operatorname{Id}_{+}(K)$ and $\cup B_{t} \in \operatorname{Id}_{+}(K)$ for any ${ }_{t} \in \operatorname{Id}_{+}(K)$; $t \in \mathrm{T} \quad \mathrm{t} \in \mathrm{T}$

3. $\underset{t \in T}{\cap} C_{t} \in I_{-} \_(K)$ and $\underset{t \in T}{\cup} C_{t} \in I_{-} \_(K)$ for any $C_{t} \in I_{-} \_(K)$.

The proof is clear. We next consider the multiplication operation $\mathrm{A} \cdot \mathrm{B}$ on $2^{\mathrm{K}}$.

Definition 3.3: Hypersemigroup is a hypergroupoid $\mathrm{K}$ such that $(A \cdot B) \cdot C=A \cdot(B \cdot C)$ for any $A, B, C \in 2^{K}$.

If $K$ is hypersemigroup then $A \cdot B \in \operatorname{Id}(K)$ for any $A$, $B \in \operatorname{Id}(K)$. But there are a hypergroupoid $K$ and $A$, $B \in \operatorname{Id}(K)$ such that $A \cdot B \notin \operatorname{Id}(K)$. Therefore for any hypergroupoid $\mathrm{K}$ we define a multiplication operation of ideals as follows:

For $\mathrm{A}, \mathrm{B} \in \operatorname{Id}(\mathrm{K})$ denote by $\mathrm{A} \cdot \mathrm{B}$ the intersection of all ideals of $K$ containing the set $G=\{x \mid x=a \cdot b, a \in A$, $\mathrm{b} \in \mathrm{B}\}$

Multiplication operations on $\operatorname{Id}_{+}(\mathrm{K})$ and $\mathrm{Id}_{-}(\mathrm{K})$ are introduced similarly.

Proposition 3.4: For any hypergroupoid $K$, the lattices $\operatorname{Id}(\mathrm{K}), \mathrm{Id}_{+}(\mathrm{K}), \mathrm{Id}_{-}(\mathrm{K})$ are complete $\ell$-groupoids with respect to above multiplication operations.

Proof: We give a proof for $\operatorname{Id}(\mathrm{K})$ and the proofs for $\mathrm{Id}_{+}(\mathrm{K})$ and $\mathrm{Id} \_(\mathrm{K})$ are similar. Suppose $\mathrm{A}, \mathrm{B}_{\mathrm{t}} \in \operatorname{Id}(\mathrm{K})$, $t \in T$. It is clear that $A \cdot\left(\cup_{t \in T} B_{t}\right) \supseteq \underset{t \in T}{\cup}\left(A \cdot B_{t}\right)$
Conversely the ideal $A \cdot\left(\cup B_{t}\right)$ is the smallest ideal containing all elements $a \cdot b$, where $a \in A, b \in \underset{t \in T}{\cup} B_{t}$. Let $a, b \in A \cdot\left(\underset{t \in T}{\cup} B_{t}\right)$.

Since $b \in B_{t}$ for some $t \in T$ then $a \cdot b \in A \cdot B_{t}$. Therefore $A \cdot\left(\cup_{t \in T} B_{t}\right) \subseteq \underset{t \in T}{\cup}\left(A \cdot B_{t}\right)$

Now, we apply the definitions and designations of the prime and semiprime elements of ordered groupoids to $2^{\mathrm{K}}, \operatorname{Id}(\mathrm{K}), \mathrm{Id}_{+}(\mathrm{K})$, Id_(K). Put

$$
\begin{aligned}
& R_{G}(A)=R(A), r_{G}^{s}(A)=r^{s}(A), r_{G}^{W}(A)=r^{w}(A) \text { for } \\
& G=I d(K), A \in \operatorname{Id}(K) \\
& R_{G}(A)=R_{+}(A), r_{G}^{s}(A)=r_{+}^{s}(A), r_{G}^{W}(A)=r_{+}^{W}(A) \text { for } \\
& G=I_{+}(K), A \in \operatorname{Id}_{+}(K)
\end{aligned}
$$$$
R_{G}(A)=R_{-}(A), r_{G}^{s}(A)=r \frac{s}{-}(A), r_{G}^{W}(A)=r \frac{W}{-}(A) \text { for }
$$

$\mathrm{G}=\mathrm{Id} \_(\mathrm{K}), \mathrm{A} \in \mathrm{Id} \_(\mathrm{K})$

$R_{G}(A)=R_{0}(A), r_{G}^{s}(A)=r{ }_{0}^{s}(A), r_{G}^{W}(A)=r_{o}^{w}(A)$ for $\mathrm{G}=2^{\mathrm{K}}, \mathrm{A} \in 2^{\mathrm{K}}$.

For $A \in \operatorname{Id}(K)$ the ideal $R(A)$ will be called radical of $A$. An ideal $A$ is called radical if $A=R(A)$

Definition 3.5: An ideal $\mathrm{H}$ is maximal if $\mathrm{H} \neq \mathrm{K}$ and $\mathrm{H} \subseteq \mathrm{B} \subseteq \mathrm{K}, \mathrm{B} \in \mathrm{Id}(\mathrm{K})$ implies that $\mathrm{H}=\mathrm{B}$ or $\mathrm{B}=\mathrm{K}$.

For $\mathrm{a} \in \mathrm{K}$ denote by $[\mathrm{a}]$ the intersection of all ideals of $\mathrm{K}$ containing a.

Proposition 3.6: Let $\mathrm{K}$ be a hypergruopoid. Then any maximal ideal of $\mathrm{K}$ is prime if and only if $\mathrm{K}=\mathrm{K}^{2}$.

Proof: Let $\mathrm{K}=\mathrm{K}^{2}$ and $\mathrm{M}$ be a maximal ideal of $\mathrm{K}$. Assume that $\mathrm{A} \cdot \mathrm{B} \subseteq \mathrm{M}, \mathrm{A}, \mathrm{B} \in \operatorname{Id}(\mathrm{K})$. If $\mathrm{A} \not \subset \mathrm{B}$ and $\mathrm{B} \not \subset \mathrm{M}$ then $A \cup M=K, B \cup M=K$. Therefore

$\mathrm{K} \cdot \mathrm{K}=(\mathrm{A} \cup \mathrm{M})(\mathrm{B} \cup \mathrm{M})=\mathrm{A} \cdot \mathrm{B} \cup \mathrm{AM} \cup \mathrm{MB} \cup \mathrm{MM} \subseteq \mathrm{M} \subseteq \mathrm{K}$ by Proposition 3.4. Hence $\mathrm{M}=\mathrm{K}$. This is a contradiction. Thus $\mathrm{M}$ is prime.

Conversely, Let $K^{2} \neq K$ and $a \in K \backslash K^{2}$. We prove that $\mathrm{M}=\mathrm{K} \backslash\{\mathrm{a}\}$ is a maximal ideal of $\mathrm{K}$ and it is not prime. Let $b \in M \backslash\{a\}$. Then $h b \in M$ and $b h \in M$ for all $h \in K$. Indeed, if there is $h \in K$ such that $h b \notin M$ then $a \in h b$.

Hence $a \in K^{2}$. It is a contradiction. Thus $h b \in M$ and $b h \in M$ for any $h \in K$. It is clear that $M$ is a maximal ideal. Prove that $\mathrm{M}$ is not prime. By $\mathrm{a} \notin \mathrm{M}$ we have $[\mathrm{a}] \not \subset \mathrm{M}$. But $[\mathrm{a}] \subseteq \mathrm{K}^{2} \subseteq \mathrm{M}$. Therefore $\mathrm{M}$ is not prime.

Remark: This proposition is known for semigroups ${ }^{[5]}$.

Every sequence $\left\{x_{0}, x_{1}, \ldots, x_{n}, \ldots\right\}$, where $x_{0}=a$, $x_{n+1} \in\left[x_{n}\right]^{2}$, will be called an s-sequence of the element a.

Definition 3.7: Let $A \in \operatorname{Id}(K)$. An element $a \in K$ is strongly A-nilpotent if every s-sequence of a meets A. 
Remark: This definition is similar to the definition of the $\mathrm{n}$-sequence ${ }^{[6]}$.

Denote by $\mathrm{n}(\mathrm{A})$ the set of all strongly A-nilpotent elements of $\mathrm{K}$.

Theorem 3.8: Let $K$ be a hypergroupoid. Then for any ideal $A$ of $K$, we have $n(A)=r^{s}(A)=r^{w}(A)=R(A)$.

Proof: From the definitions $r^{\mathrm{s}}(\mathrm{A}), \mathrm{r}^{\mathrm{w}}(\mathrm{A}), \mathrm{R}(\mathrm{A})$ we obtain $r^{\mathrm{s}}(A) \subseteq r^{\mathrm{w}}(A) \subseteq R(A)$ for any $A \in \operatorname{Id}(K)$.

We prove that $n(A) \subseteq r^{\mathrm{s}}(A)$. If there is not an ssemiprime ideal of $K$ containing $A$ then $r^{\mathrm{s}}(A)=K$ and $\mathrm{n}(\mathrm{A}) \subseteq \mathrm{r}^{\mathrm{s}}(\mathrm{A})$.

Assume that there exists an s-semiprime ideal of $\mathrm{K}$ containing A. Let $\mathrm{a} \in \mathrm{n}(\mathrm{A})$ and $\mathrm{S}$ be s-semiprime ideal of $\mathrm{K}$ containing $\mathrm{A}$. We first prove that $\mathrm{a} \in \mathrm{S}$. If $\mathrm{a} \notin \mathrm{S}$, then $\left[x_{0}\right] \not \subset S$, where $x_{0}=a$. There exists $x_{1} \in\left[x_{0}\right]^{2}$ such that $\mathrm{x}_{1} \notin \mathrm{S}$ since $\left[\mathrm{x}_{\mathrm{o}}\right]^{2} \not \subset \mathrm{S}$.

By continuing in this manner we obtain an ssequence $\left\{\mathrm{x}_{0}, \mathrm{x}_{1}, \ldots, \mathrm{x}_{\mathrm{n}}, \ldots\right\}$ of the element a such that $\mathrm{x}_{\mathrm{n}} \notin \mathrm{S}$ for all $\mathrm{n}$. But this is a contradiction since every ssequence of the element a meets $A$. Thus $a \in S$ and $a \in r^{s}(A)$ since $S$ is any semiprime ideal containing $A$. Hence $\mathrm{n}(\mathrm{A}) \subseteq \mathrm{r}^{\mathrm{s}}(\mathrm{A}) \subseteq \mathrm{r}^{\mathrm{w}}(\mathrm{A}) \subseteq \mathrm{R}(\mathrm{A})$.

Now we prove that $R(A)=n(A)$. If $n(A)=K$ then $n(A)=r^{\mathrm{s}}(A)=r^{\mathrm{w}}(A)=R(A)=K$. Let $n(A) \neq K$. Hence there exists $b \in K$ such that $b \notin n(A)$. Then there exists an $s-$ sequence $X=\left\{x_{0}, x_{1}, \ldots, x_{n}, \ldots\right\}$ of the element $b$ such that $\mathrm{X} \cap \mathrm{A}=\varnothing$. Denote by $\Sigma$ the set of ideals $\mathrm{M}$ in $\mathrm{K}$ such that $\mathrm{X} \cap \mathrm{M}=\varnothing, \mathrm{M} \supseteq \mathrm{A}$. $\Sigma$ is not empty since $\mathrm{A} \in \Sigma$.

We can apply Zorn's lemma to the set $\Sigma$ so there exists a maximal element $\mathrm{P}$ of $\Sigma$. We show that $\mathrm{P}$ is prime.

First, $P$ is proper since $b \notin P$. Let $B, C \in \operatorname{Id}(K), B \not \subset P$, $\mathrm{C} \not \subset \mathrm{P}$. Then $\mathrm{P} \cup \mathrm{B} \neq \mathrm{P}$ and $\mathrm{P} \cup \mathrm{C} \neq \mathrm{P}$. By the maximality of $\mathrm{P}$ in $\Sigma$. We have $\mathrm{P} \cup \mathrm{B} \notin \Sigma$ and $\mathrm{P} \cup \mathrm{C} \notin \Sigma$. Hence there exist $x_{m} \in X, x_{q} \in X$ such that $x_{m} \in P \cup B, x_{q} \in P \cup C$. Then $\left[x_{m}\right] \subseteq P \cup B, \quad\left[x_{q}\right] \subseteq P \cup C$. Hence $\quad x_{m+1} \in\left[x_{m}\right]^{2} \subseteq P \cup B$, $\mathrm{x}_{\mathrm{q}+1} \in\left[\mathrm{x}_{\mathrm{q}}\right]^{2} \subseteq \mathrm{P} \cup \mathrm{C}$. By continuing in this manner we find $x_{m+1} \in P \cup B, x_{q+1} \in P \cup C$ for all t. Put $n=\max (m, q)$. Then $x_{n} \in P \cup B, \quad x_{n} \in P \cup C$. Hence, $\quad x_{n+1} \in\left[x_{n}\right]^{2} \subseteq(P \cup B)$. $(\mathrm{P} \cup \mathrm{C}) \subseteq \mathrm{P} \cup \mathrm{B} \cdot \mathrm{C}$ by the Proposition 3.4. But $\mathrm{x}_{\mathrm{n}+1} \notin \mathrm{P}$. Hence $B \cdot C \not \subset P$. Therefore $P$ is prime. Thus there exists a prime ideal $P$ such that $b \notin P$. Thus $\mathrm{n}(\mathrm{A})=\mathrm{r}^{\mathrm{s}}(\mathrm{A})=\mathrm{r}^{\mathrm{w}}(\mathrm{A})=\mathrm{R}(\mathrm{A})$. From the Theorem 3.8, we obtain that every s-semiprime ideal of $\mathrm{K}$ is radical.

The ideal $\mathrm{R}(\varnothing)$ will be called the prime radical of the hypergroupoid $\mathrm{K}$ and will be denoted by $\operatorname{Pr} . \operatorname{rad}(\mathrm{K})$.

Corollary 3.9: For any ideal A of $\mathrm{K}$ the following conditions are equivalent:

1. $\mathrm{R}(\mathrm{A})=\mathrm{A}$;

2. If $\mathrm{B}^{(\mathrm{n})} \subseteq \mathrm{A}, \mathrm{B} \in \mathrm{Id}(\mathrm{K})$, for some $\mathrm{n}$ then $\mathrm{B} \subseteq \mathrm{A}$.

3. If $\mathrm{B}^{2} \subseteq \mathrm{A}, \mathrm{B} \in \operatorname{Id}(\mathrm{K})$, then $\mathrm{B} \subseteq \mathrm{A}$.
Proof: $(1) \Rightarrow(2) \Rightarrow(3)$ is clear. (3) $\Rightarrow(2)$ : Let $\mathrm{B}^{(\mathrm{n})} \subseteq \mathrm{A}$, $\mathrm{B} \in \operatorname{Id}(\mathrm{K})$, for some $\mathrm{n}$. Then $\mathrm{B}^{(\mathrm{n})}=\left(\mathrm{B}^{(\mathrm{n}-1)}\right)^{2} \subseteq \mathrm{A}$ implies that $\mathrm{B}^{(\mathrm{n}-1)} \subseteq \mathrm{A}$. By induction on $\mathrm{n}$ we obtain $\mathrm{B} \subseteq \mathrm{A}$. $(2) \Rightarrow(1)$ : The condition (2) implies that $\mathrm{r}^{\mathrm{s}}(\mathrm{A})=\mathrm{A}$. By the Theorem 3.8 we see $\mathrm{R}(\mathrm{A})=\mathrm{r}^{\mathrm{s}}(\mathrm{A})=\mathrm{A}$.

Corollary 3.10: For a hypergropoid $\mathrm{K}$ the following conditions are equivalent:

1. Every ideal of $\mathrm{K}$ is radical;

2. $\mathrm{A} \cdot \mathrm{B}=\mathrm{A} \cap \mathrm{B}$ for all $\mathrm{A}, \mathrm{B} \in \operatorname{Id}(\mathrm{K})$;

3. $[\mathrm{a}]^{2}=[\mathrm{a}]$ for all $\mathrm{a} \in \mathrm{K}$.

Proof: We use the following lemma:

Lemma 3.11: $R(A \cdot B)=R(A \cap B)=R(A) \cap R(B)$ for any $\mathrm{A}, \mathrm{B} \in \operatorname{Id}(\mathrm{K})$.

The proof of this lemma follows from the Proposition $1.6^{[7]}$.

$(1) \Rightarrow(2)$ : If every ideal of $K$ is radical then using the lemma we obtain

$A \cdot B=R(A \cdot B)=R(A) \cap R(B)=A \cap B . \quad(2) \Rightarrow(3)$ : $\quad$ Let $A \cdot B=A \cap B$ for all $A, B \in \operatorname{Id}(K)$. Then $A^{2}=A$ for all $A \in \operatorname{Id}(K)$. (3) $\Rightarrow(1)$ : We prove that every ideal of $K$ is ssemiprime. Let $\mathrm{A}$ be an ideal of $\mathrm{K}$. Then $\mathrm{A}=\underset{a \in A}{\cup}[\mathrm{a}]$. Using the Proposition 3.4 we have $\mathrm{A}^{2}=(\underset{\mathrm{a} \in \mathrm{A}}{\cup}[\mathrm{a}])^{2}=\left(\cup_{a \in A}[\mathrm{a}]^{2}\right) \cup\left(\cup_{a \in A}[\mathrm{a}][\mathrm{b}]\right)=\cup_{a \in A}[\mathrm{a}]=\mathrm{A}$ since $[a] \cdot[b] \subseteq[a] \cap[b]$ for any $a, b \in A$. Thus $A^{2}=A$ for all $\mathrm{A} \in \operatorname{Id}(\mathrm{K})$. Assume that $\mathrm{B}^{2} \subseteq \mathrm{A}, \quad \mathrm{B} \in \operatorname{Id}(\mathrm{K})$. Then $\mathrm{B}=\mathrm{B}^{2} \subseteq \mathrm{A}$. Therefore $\mathrm{A}$ is s-semiprime. From the Theorem 3.8 we obtain that $\mathrm{A}$ is radical.

Remark: This corollary is an analog of the similar theorem for associative rings ${ }^{[8]}$.

Definition 3.12: Let $A \in \operatorname{Id}(K)$. An ideal $B$ of $K$ is $A_{s}$ nilpotent if $\mathrm{B}^{(\mathrm{n})} \subseteq \mathrm{A}$ for some $\mathrm{n}$.

Proposition 3.13: Let $\mathrm{K}$ be hypergroupoid and $\mathrm{A}$, $\mathrm{B} \in \operatorname{Id}(\mathrm{K})$. If $\mathrm{C}$ is $\mathrm{B}_{\mathrm{s}}$-nilpotent and $\mathrm{B}$ is $\mathrm{A}_{\mathrm{s}}$-nilpotent then $\mathrm{C}$ is $\mathrm{A}_{\mathrm{s}}$-nilpotent.

Proof: Since $\mathrm{C}$ is $\mathrm{B}_{\mathrm{s}}$-nilpotent then $\mathrm{C}^{(\mathrm{n})} \subseteq \mathrm{B}$ for some $\mathrm{n}$. Hence $C^{(n+m)}=\left(C^{(n)}\right)^{(m)} \subseteq B^{(m)} \subseteq$ A for some $m$.

Theorem 3.14: Let $\mathrm{K}$ be a hypergroupoid satisfying the ascending chain condition for ideals. Then for any ideals $\mathrm{A}$ of $\mathrm{K}, \mathrm{R}(\mathrm{A})$ is $\mathrm{A}_{\mathrm{s}}$-nilpotent.

Proof: Let $A \in \operatorname{Id}(K)$. Denote by $\Sigma$ the set of all $A_{s}-$ nilpotent ideals $H$ of $K$ such that $H \supseteq A$. $\Sigma$ is not empty since $A \in \Sigma$. There exists a maximal element $P$ in $\Sigma$. We prove that $P$ is s-semiprime. Let $B^{2} \subseteq P$. Then $(\mathrm{B} \cup \mathrm{P})^{2}=\mathrm{B}^{2} \cup \mathrm{BP} \cup \mathrm{PB} \cup \mathrm{P}^{2} \subseteq \mathrm{P}$. By Proposition 3.13 the 
ideal $B \cup P$ is $A_{s}$-nilpotent. By the maximality of $P$ we have $B \cup P=P$. Hence $B \subseteq P$. This means that $P$ is $s-$ semiprime. Since $\mathrm{P} \supseteq \mathrm{A}$ then $\mathrm{R}(\mathrm{A}) \subseteq \mathrm{P}$ by Theorem 3.8. But $P^{(n)} \subseteq A \subseteq R(A)$ for some $n$. Since $R(A)$ is $s-$ semiprime then $P \subseteq R(A)$. Thus $P=R(A)$

Remark: This theorem is similar to the proposition for associative rings ${ }^{[9]}$.

Corollary 3.15: Let $\mathrm{K}$ be hypergroupoid satisfying the ascending chain condition for ideals. Then the following conditions are equivalent:

1. $\mathrm{K}^{\mathrm{(n)}}=\varnothing$ for some $\mathrm{n}$.

2. K doesn't have a prime ideal;

3. K doesn't have a s-semiprime ideal.

A proof follows from Theorem 3.14 and the definition of $\operatorname{Pr} . \operatorname{rad}(\mathrm{K})$. Denote by $\operatorname{Id}_{\mathrm{r}}(\mathrm{K})$ the set of all radical ideals of $\mathrm{K}$. $\operatorname{Id}_{\mathrm{r}}(\mathrm{K})$ is a complete lattice with respect to the inclusion relation. Denote by $\vee$ and $\wedge$ the lattice operations in $\operatorname{Id}_{\mathrm{r}}(\mathrm{K})$.

Theorem 3.16: Let $\mathrm{K}$ be a hypergroupoid. Then the lattice $\operatorname{Id}_{\mathrm{r}}(\mathrm{K})$ satisfies the infinite $\wedge$-distributive condition:

$\mathrm{A} \wedge\left(\underset{t \in T}{\vee} B_{t}\right)=\vee\left(\underset{t \in T}{A \wedge} \wedge B_{t}\right)$ for any $\mathrm{A}, \mathrm{B}_{\mathrm{t}} \in \mathrm{Id}_{\mathrm{r}}(\mathrm{K})$

Proof: The proof follows from Theorem $1.3^{[7]}$.

Theorem 3.17: Let $\mathrm{K}$ be a hypergroupoid satisfying the ascending chain candition for ideals. Then any radical ideal of $\mathrm{K}$ is an intersection of finite prime ideals and a such representation is unique.

Proof: First we prove the following lemma.

Lemma: $\mathrm{H} \in \mathrm{Id}_{\mathrm{r}}(\mathrm{K})$ is prime ideal if and only if $\mathrm{H}$ is an $\wedge$-indecomposable element of the lattice $\operatorname{Id}_{\mathrm{r}}(\mathrm{K})$.

Proof: Let $A$ be a prime ideal of $K$ and $A=A_{1} \wedge A_{2}, A_{1}$, $\mathrm{A}_{2} \in \mathrm{Id}_{\mathrm{r}}(\mathrm{K})$. Then ${ }^{[7]}$.

$$
A_{1} A_{2} \subseteq A_{1} \cap A_{2} \subseteq R\left(A_{1} \cap A_{2}\right)=A_{1} \wedge A_{2}=A \text {. Hence }
$$

$A_{1} \subseteq A$ or $A_{2} \subseteq A$. Then $A=A_{1}$ or $A=A_{2}$ Let $A$ be an $\wedge^{-}$ indecomposable element in $\operatorname{Id}_{\mathrm{r}}(\mathrm{K})$ and $\mathrm{BC} \subseteq \mathrm{A}, \mathrm{B}$, $\mathrm{C} \in \operatorname{Id}(\mathrm{K})$. Then $\mathrm{R}(\mathrm{B} \cdot \mathrm{C}) \subseteq \mathrm{A}$. By the lemma $1.6^{[7]}$ we have $R(B) \wedge R(C)=R(B \cdot C) \subseteq A$. By the distributivity $\operatorname{Id}_{r}(K)$ we obtain $A=A \vee(R(B) \wedge R(C))=(A \vee R(B)) \wedge$ $(A \vee R(C))$. Then $A=A \vee R(B)$ or $A=A \vee R(C)$ since $A$ is $\wedge$-indecomposable. This means that $\mathrm{B} \subseteq \mathrm{R}(\mathrm{B}) \subseteq \mathrm{A}$ or $\mathrm{C} \subseteq \mathrm{R}(\mathrm{C}) \subseteq \mathrm{A}$.

Thus A is prime. The lemma is proved. By the lemma and the Corollary ${ }^{[1]}$ we obtain that every radical ideal of $\mathrm{K}$ is an intersection of finite prime ideals and a such represantation is unique.

\section{The Right Prime Radical of an Ideal \\ Definition 4.1: A right ideal $H$ of $K$ is maximal if $H \neq K$ and $\mathrm{H} \subseteq \mathrm{B} \subseteq \mathrm{K}, \mathrm{B} \in \mathrm{Id}_{+}(\mathrm{K})$, implies that $\mathrm{H}=\mathrm{B}$ or $\mathrm{B}=\mathrm{K}$.}

Proposition 4.2: Let $\mathrm{K}$ be a hypergroupoid such that $\mathrm{A} \subseteq \mathrm{K} \cdot \mathrm{A}$ for all $\mathrm{A} \in \mathrm{Id}_{+}(\mathrm{K})$. Then any maximal right ideal of $K$ is prime element of $\operatorname{Id}_{+}(K)$.

Proof: Let $\mathrm{M}$ be a maximal right ideal of $\mathrm{K}$ and $A \cdot B \subseteq M, A, B \in I_{+}(K)$. If $A \not \subset M$ then $M \cup A=K$. $B y$ Proposition 3.4 we have $\mathrm{B} \subseteq \mathrm{K} \cdot \mathrm{B}=(\mathrm{M} \cup \mathrm{A}) \cdot \mathrm{B}=\mathrm{MB} \cup \mathrm{AB} \subseteq \mathrm{M}$.

Definition 4.3: An element $1 \in K$ is called identity of $K$ if $1 \cdot a=a \cdot 1=a$ for all $a \in K$.

Remark: The conditions of Proposition 4.2 are satisfied for groupoids with 1 . Thus there exists a prime right ideal in such groupoids.

For an element $\mathrm{a} \in \mathrm{K}$ denote by $[\mathrm{a}]_{+}$the intersection of all right ideals containing a. Every sequence $\left\{\mathrm{x}_{0}, \ldots\right.$, $\left.\mathrm{x}_{\mathrm{n}}, \ldots.\right\}$, where $\mathrm{x}_{0}=\mathrm{a}, \mathrm{x}_{\mathrm{m}+1} \in\left[\mathrm{x}_{\mathrm{m}}\right]_{+}^{2}$, is called an $\mathrm{s}_{+^{-}}$ sequence of the element a.

Definition 4.4: Let $A \in \operatorname{Id}_{+}(K)$. An element $a \in K$ is strongly $\mathrm{A}_{+}$-nilpotent if every its $\mathrm{s}_{+}$-sequence meets $\mathrm{A}$.

Denote by $n_{+}(A)$ the set of all strongly $A_{+}$-nilpotent elements of $\mathrm{K}$.

Proposition 4.5: Let $\mathrm{K}$ be a hypergroupoid. For any right ideal $\mathrm{A}$ of $\mathrm{K}$ are satisfied the following inequalities:

$\mathrm{R}(\mathrm{A}) \subseteq \mathrm{n}_{+}(\mathrm{A}) \subseteq \mathrm{r}_{+}^{\mathrm{s}}(\mathrm{A}) \subseteq \mathrm{r}_{+}^{\mathrm{W}}(\mathrm{A}) \subseteq \mathrm{R}_{+}(\mathrm{A})$.

Proof: A proof of $n_{+}(A) \subseteq r_{+}^{s}(A)$ is similar to the proof of $n(A) \subseteq r^{\mathrm{s}}(A)$ as in the Theorem 3.8. The inequality $R(A) \subseteq n_{+}(A)$ immediately follows from the equality $\mathrm{R}(\mathrm{A})=\mathrm{n}(\mathrm{A})$ and definitions of $\mathrm{n}(\mathrm{A})$ and $\mathrm{n}_{+}(\mathrm{A})$.

Theorem 4.6: Let $\mathrm{K}$ be a hypergroupoid satisfying the following conditions:

$(\mathrm{K} \cdot \mathrm{A}) \cdot \mathrm{B}=\mathrm{K} \cdot(\mathrm{A} \cdot \mathrm{B}), \quad(\mathrm{A} \cdot \mathrm{K}) \cdot \mathrm{B}=\mathrm{A} \cdot(\mathrm{K} \cdot \mathrm{B}) \quad$ for $\quad$ all $\quad \mathrm{A}$, $\mathrm{B} \in \mathrm{Id}_{+}(\mathrm{K})$. Then

$R(A)=n_{+}(A)=r \stackrel{s}{s}(A)=r_{+}^{w}(A)=R_{+}(A)$ for any $A \in \operatorname{Id}(K)$.

Proof: By Proposition 4.5 it is enough to prove that $R_{+}(A) \subseteq R(A)$.

Denote by $\mathrm{P}(\mathrm{K})$ the set of all prime ideals of $\mathrm{K}$ and by $\mathrm{P}_{+}(\mathrm{K})$ the set of all prime right ideals of $\mathrm{K}$. We prove that $\mathrm{P}(\mathrm{K}) \subseteq \mathrm{P}_{+}(\mathrm{K})$. Let $\mathrm{Q} \in \mathrm{P}(\mathrm{K})$ and $\mathrm{B} \cdot \mathrm{C} \subseteq \mathrm{Q}, \quad \mathrm{B}$, $\mathrm{C} \in \mathrm{Id}_{+}(\mathrm{K})$. Then, $(\mathrm{B} \cup \mathrm{K} \cdot \mathrm{B})(\mathrm{C} \cup \mathrm{K} \cdot \mathrm{C})=(\mathrm{B} \cdot \mathrm{C}) \cup(\mathrm{B} \cdot(\mathrm{KC}))$ $\cup((\mathrm{K} \cdot \mathrm{B}) \cdot \mathrm{C}) \cup(\mathrm{K} \cdot \mathrm{B}) \cdot(\mathrm{K} \cdot \mathrm{C}) \subseteq \mathrm{Q}$.

Note that $B \cup K B$ and $C \cup K C$ are ideals of $K$. Indeed $\mathrm{K} \cdot(\mathrm{B} \cup \mathrm{KB})=\mathrm{K} \cdot \mathrm{B} \cup(\mathrm{K} \cdot(\mathrm{K} \cdot \mathrm{B})) \subseteq \mathrm{B} \cup \mathrm{KB}$.

From $\quad(B \cup K B) \quad(C \cup K C) \subseteq Q$ we obtain $\mathrm{B} \subseteq \mathrm{B} \cup \mathrm{KB} \subseteq \mathrm{Q}$ or $\mathrm{C} \subseteq \mathrm{C} \cup \mathrm{KC} \subseteq \mathrm{Q}$ since $\mathrm{Q}$ is prime. This means $Q \in \mathrm{P}_{+}(\mathrm{K})$. 
Thus $\mathrm{P}(\mathrm{K}) \subseteq \mathrm{P}_{+}(\mathrm{K})$. Therefore we have $\mathrm{R}_{+}(\mathrm{A}) \subseteq \mathrm{R}(\mathrm{A})$.

Remark: The conditions of this theorem are satisfied for hypersemigroup. Therefore the same theorem is given for nonasociative hypergroupoid $K$ and $A \in \operatorname{Id}(K)$ such that $\mathrm{R}(\mathrm{A})=\mathrm{R}_{+}(\mathrm{A})$ and $\mathrm{R}(\mathrm{A}) \neq \mathrm{R}_{-}(\mathrm{A})$.

Let $A \in \operatorname{Id}_{+}(K)$. For $b \in K$ put $b^{(0)}=b, b^{(n+1)}=\left(b^{(n)}\right)^{2}$.

Definition 4.7: An element $b \in K$ is $A_{s}$-nilpotent if $b^{(n)} \subset A$ for some $n$. An element $b \in K$ is $A_{w}$-nilpotent if $\mathrm{f}(\mathrm{b}) \subseteq \mathrm{A}$ for some $\mathrm{f}(\mathrm{b}) \subseteq<\mathrm{b}>$.

Denote by $\mathrm{n}_{\mathrm{O}}^{\mathrm{s}}(\mathrm{A})\left(\mathrm{n}_{\mathrm{O}}^{\mathrm{W}}(\mathrm{A})\right)$ the set of all $\mathrm{A}_{\mathrm{s}}$ nilpotent (respectively, $A_{w}$-nilpotent) elements of $\mathrm{K}$.

Proposition 4.8: For any ideal A of $\mathrm{K}$ are hold the following inequalities:

$\mathrm{R}(\mathrm{A}) \subseteq \mathrm{n}_{+}(\mathrm{A}) \subseteq \mathrm{n}_{\mathrm{O}}^{\mathrm{s}}(\mathrm{A}) \subseteq \mathrm{r}_{\mathrm{O}}^{\mathrm{s}}(\mathrm{A}) \subseteq \mathrm{R}_{\mathrm{o}}(\mathrm{A})$

$\mathrm{R}(\mathrm{A}) \subseteq \mathrm{n}_{+}(\mathrm{A}) \subseteq \mathrm{n}_{\mathrm{O}}^{\mathrm{W}}(\mathrm{A}) \subseteq \mathrm{r}_{\mathrm{O}}^{\mathrm{W}}(\mathrm{A}) \in \mathrm{R}_{\mathrm{o}}(\mathrm{A})$

The proof is smilar to the proof of Proposition 4.5.

Theorem 4.9: Let $K$ be a hypersemigroup satisfying the condition $\mathrm{K} \cdot \mathrm{a}=\mathrm{a} \cdot \mathrm{K}$ for all $\mathrm{a} \in \mathrm{K}$. Then $R(A)=n_{0}(A)=r_{0}(A)=R_{0}(A)$ for all $A \in \operatorname{Id}(K)$.

The proof is smilar to the proof of Theorem 4.6.

\section{REFERENCES}

1. Birkhoff, G., 1967. Lattice Theory, Providence, Rhode Island.

2. Levy-Bruhl, J., 1968. Introduction Aux Structures Algebriques, Dunod, Paris.

3. Khadjiev, D. and F. Çallıalp, 1997. On the prime radical of a ring and a groupoid. Marmara Univ. Fen Dergisi, no. 13, İstanbul.

4. Clifford, A.H. and G.B. Preston, 1967. The Algebraic Theory of Semigroups. Vol. I, Providence, Rhode Island.

5. Artamonov, V.A., V.N. Saliy, L.A. Skornyakov, L.N. Shevrin and E.G. Shulgeyfer, 1991. General Algebra, T.2. Moskow, Nauka.

6. Lambek, J., 1966. Lectures on Rings and Modules. Blaisdell Publ., Comp., Waltham-London.

7. Khadjiev, D.J. and T.M. Shamilev, 1997. Complete $\ell$-groupoids and their prime spectrums. Algebra i Logica (Algebra and Logic), 86: 341-355.

8. Andrunakievich, V.A. and Y.M. Ryabuhin, 1979. Radicals of Algebras and a Structure Theory. Moscow, Nauka.

9. Rowen, L.H., 1988. Ring Theory. Vol.1, Acad. Press. INC., Boston. 